\title{
Japan bids to form new centres of excellence
}

Tokyo. Japan's Science and Technology Agency (STA) will pump \$20 million of extra funds over the next five years into each of two national research institutes in the hope of converting them into "centres of excellence' (COEs) able to compete with the world's leading research institutions.

The two institutes, whose names were announced last week, are the National Institute of Agrobiological Resources of the Ministry of Agriculture, Forestry and Fisheries, and the Communications Research Laboratory of the Ministry of Posts and Telecommunications.

They join three other institutes chosen as potential centres of excellence last year (see Nature 364, 660; 1993). But critics say it will take a lot more than just money to bring about the change.

Japan's centre-of-excellence scheme is unique among such government-funded schemes around the world in that money is directed not at institutes already judged to conduct research of a high standard, but rather those judged capable of doing so in the future. One result of this policy is that some of Japan's best research institutes stand little chance of getting an award.

In theory, more than a hundred national research institutes and semi-government research organizations affiliated to Japan's various ministries and agencies could apply for $\mathrm{COE}$ funding (those affiliated to the Ministry of Education, Science and Culture are excluded from consideration).

In practice, however, comparatively few institutes have officially applied. This year, for example, only 12 institutes did so, compared with 19 in the first year of operation of the scheme. The two selected were chosen after they had been screened by a committee of 17 leading academics and industrialists, headed by Wataru Mori of the Council of Science and Technology, Japan's leading policy-making science body.

Most institutes are eliminated at an earlier stage by unofficial internal screening within the various ministries and agencies. For example, Japan's well-known Institute of Physical and Chemical Research (RIKEN) was hoping to apply this year.

RIKEN officials had planned to seek funds to reinforce some of its weaker divisions. But they were informally told by STA that this idea did not fit its strategy of supporting institutes as a whole, and subsequently decided not to proceed with their application.

Similarly, the National Cancer Center Research Institute has held back from applying for COE funds because it is in the middle of negotiations for funds for a new
10 year cancer programme.

STA officials insist that external factors do not influence the selection of institutes for COE funding, and that any institute can apply. But they do admit that each ministry and agency probably decides through its own internal processes which of its institutes it will put forward for COE funding.

A researcher at one of the five institutes already selected says he is sceptical that the COE funds will achieve their goal of nurturing excellence. Extra money helps, he admits, but will not in itself address fundamental problems in the management and administration of many national institutes.

He says the heads of institutes are often out of touch with the latest developments in science. And, with the exception of a few institutes, such as RIKEN, there is no process of external review of institutes. As a result, most researchers are not doing firstrate research, yet they are in a position to influence the decision-making of the institute head and its management.

A review of each of the COE-funded institutes will be carried out in the third year of their five-year term of funding by the committee that selected the institutes. But no external review by scientists unrelated to the programme is planned.

David Swinbanks

\section{Citation analysis confirms Australian science's declining influence}

Sydney. The quality of science in Australia relative to that elsewhere seems to have fallen markedly since the mid-1980s, according to a detailed analysis of the number and citation rate of papers produced by Australian scientists and published last week.

Paul Bourke and Linda Butler, of the Performance Indicators Project at the Australian National University's Research School of Social Sciences, found that Australians still produce about the same proportion of the world's research papers - about two per cent - as in 1987.

But, building on a theme first raised last year by the US Institute for Scientific Information (ISI) in its publication Science Watch - and following similar work on British science carried out at the University of Sussex's Science Policy Research Unit - they also found that the citation rate had fallen by 25 per cent over the same period.

The breakdown of publication and citation rates by disciplines in the analysis of ISI data reveals some large variations.

For example, Australia has managed to maintain its long-standing prominence in agriculture and earth sciences. Similarly, the citation rate remained reasonably stable in medical science, industrial biotechnology and food sciences.

But citation rates in the non-medical biological sciences, in physics and in chemistry have each fallen below the international levels they had reached in the mid1980 s. Even more worrying, citation rates dropped by 41 per cent in information, computing and communication technologies.

A comparison of the results with the performance of other countries in the ISI analysis showed that only one country (Sweden) has a similar pattern of producing as much research as a decade ago but being quoted less widely.

Canada, Germany, Japan, the Netherlands, China and South Korea are each producing more research, and this is being cited more often. Researchers in France and Switzerland are contributing a stable proportion of papers, but they are earning more citations.

Bourke points out that countries in which citation rates have been declining - namely Australia, Sweden and Britain - are also those in which a relatively high proportion of research is performed in universities. But he emphasizes that a lot of work is still needed before the reasons for the decline can be properly identified.

He speculates that possible reasons could include a general shift from basic to applied research - a major theme emphasized in government funding for science - the ageing of the Australian scientific community and a decaying research infrastructure.

Mark Lawson
While Australia reflects on the declining impact of its science output, figures just released by the ISI (right) reveal that its Pacific Rim neighbours are enjoying a steady increase in the number of papers published and cited. Singapore in particular has seen a surge in papers published from 167 papers in 1981 to 1,220 in 1993.

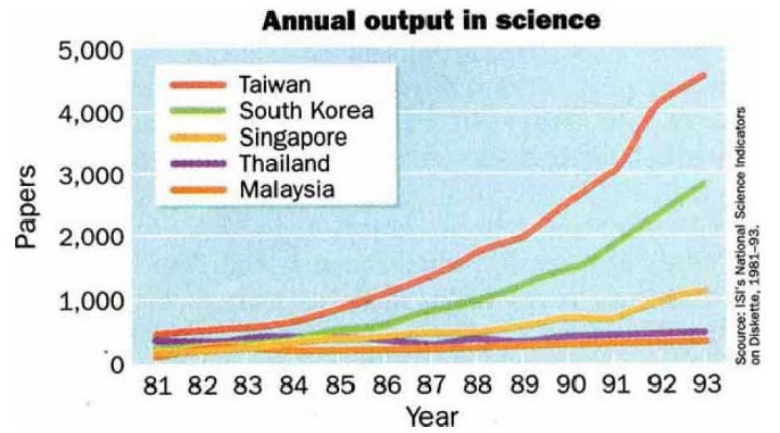

